\title{
Single fibre electromyographic studies in myasthenia gravis with repetitive nerve stimulation
}

\author{
MARTIN S. SCHWARTZ AND ERIK STÅLBERG \\ From the Department of Clinical Neurophysiology, Academic Hospital, Uppsala, Sweden
}

SYNOPSIS A method is described for repetitive stimulation studies in myasthenia gravis using submaximal stimulation and single muscle fibre recording techniques. It was found that there were no impulse blockings due to neuromuscular transmission factors in normal subjects with $2 \mathrm{~Hz}$ stimulation, although there was a decrease or increase in the number of potentials which was caused by axonal stimulation factors. In myasthenia gravis a pathological picture was obtained, consisting of impulse blockings and facilitation at this rate in all of the eight patients studied, even those with only the ocular form of myasthenia and without surface decrement in the ADM. This technique allows $\dot{\omega}_{\infty}$ study of both the minimally involved motor endplates and those with pronounced neuromuscular disturbances.

The method of single fibre electromyography (SFEMG) has been used for analysis of the neuromuscular transmission in myasthenia gravis (Ekstedt and Stålberg, 1967; Stålberg et al., 1974) both for diagnosis and evaluation of therapy. The minimal functional disturbance as recorded with this technique is an increased jitter. In the more severe transmission defects, the jitter is further increased and impulse blockings occur. When the transmission defect is very pronounced in the individual motor endplate, impulse blockings occur after only a few discharges and long-lasting impulse blockings may appear before an acceptable recording is possible. Thus a recording may not be made from the most affected motor endplates, because several discharges of the potential pairs are required before an acceptable recording for quantitative measurements is possible. In such cases the normal and less affected motor endplates may be preferentially recorded and there is a bias towards the more normal neuromuscular junctions.

It was of interest to establish a technique with the selectivity of the voluntary SFEMG recording using electrical stimulation to study both the clinically uninvolved muscles and those with

(Accepted 19 February 1975.) severe dysfunction in order to have a more accurate profile of the overall neuromusculat status. In a number of patients showing clear abnormal neuromuscular transmission, as eve denced by increased jitter and occasional in pulse blockings with SFEMG, routine tests with repetitive stimulation of the nerve to the same muscle investigated-for example, abductor digiti minimi-may be within normal limits. The relationship between the voluntary single fibre electromyographic studies and the decremental response with both surface and single fibre recording was also studied.

\section{METHODS}

The SFEMG voluntary recordings were made with a $25 \mu \mathrm{m}$ electrode in the side of a $0.5 \mathrm{~mm}$ steel cannula with moderate voluntary activation (Ekstedt, 1964; Stålberg and Ekstedt, 1973) both in the abductor 0 digiti minimi muscle (ADM) and in the extensor digitorum communis muscle (EDC).

Surface electrode recordings were performed from the ADM at supramaximal neural stimulation with $\tilde{N}$ surface stimulating electrodes at the wrist using a $N$ Medelec MS 6 electromyograph with stimulation N unit NS 6. The decremental response at $2 \mathrm{~Hz}$ and $10 \mathrm{\omega}$ $\mathrm{Hz}$ stimulation was measured in the standard manner. 
recordings made with an SFEMG electrode in the same muscle. Stimulation strength was set to submaximal levels where several distinct spikes were evoked. The stimulation was performed at $2 \mathrm{~Hz}$ usually for four stimuli but long trains were also

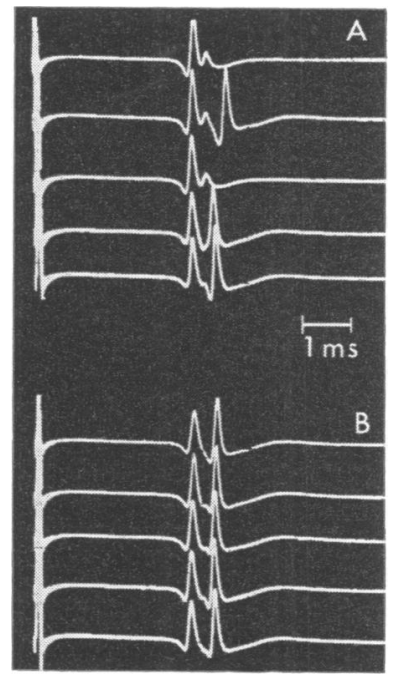

FIG. 1 The effect of submaximal axonal stimulation in normal subjects. In A there is a variation in latency and blocking on two occasions of the second large component. In B with increased stimulation strength there is a consistent response with a similar latency. made. Occasionally, responses to 5 and $10 \mathrm{~Hz}$ stimuli were also studied. Three sets of trains of $2 \mathrm{~Hz}$ stimuli were made from each of six or more different recording positions. The stimulating electrode was changed slightly once halfway through each investigation. In order to determine whether critical stimulation caused some of the observed blockings or spike recruitments each visually recognized abnormal train was further tested at a higher and lower strength of stimulation (Fig. 1). When stimulation factors were recognized to cause impulse failure or facilitation, the preceding train was excluded. However, because of the large number of components in many recordings, possible stimulation factors may have gone undetected at the experimental session.

The recordings were displayed on ultravioletsensitive (UV) film directly or after replaying the analogue tape (AKAI X330) on which all the recordings were stored. The distinct spike components generated by single muscle fibres in the first and fourth response was particularly noted. The jitter was studied usually by superimpositions of photographs. Ordinary jitter recordings with time-interval measurements could be performed only when a few non-interfering spike components were recorded. The responses were usually analysed by hand from UV film and some by electronic subtraction of the fourth from the first response. The total number of spikes was also summed for the whole experiment for the first and fourth response respectively and the results were expressed as percentage change in the number of spikes for the fourth response in relationship to the first. In addition, changes of the spike

TABLE

SFEMG STIMULATION

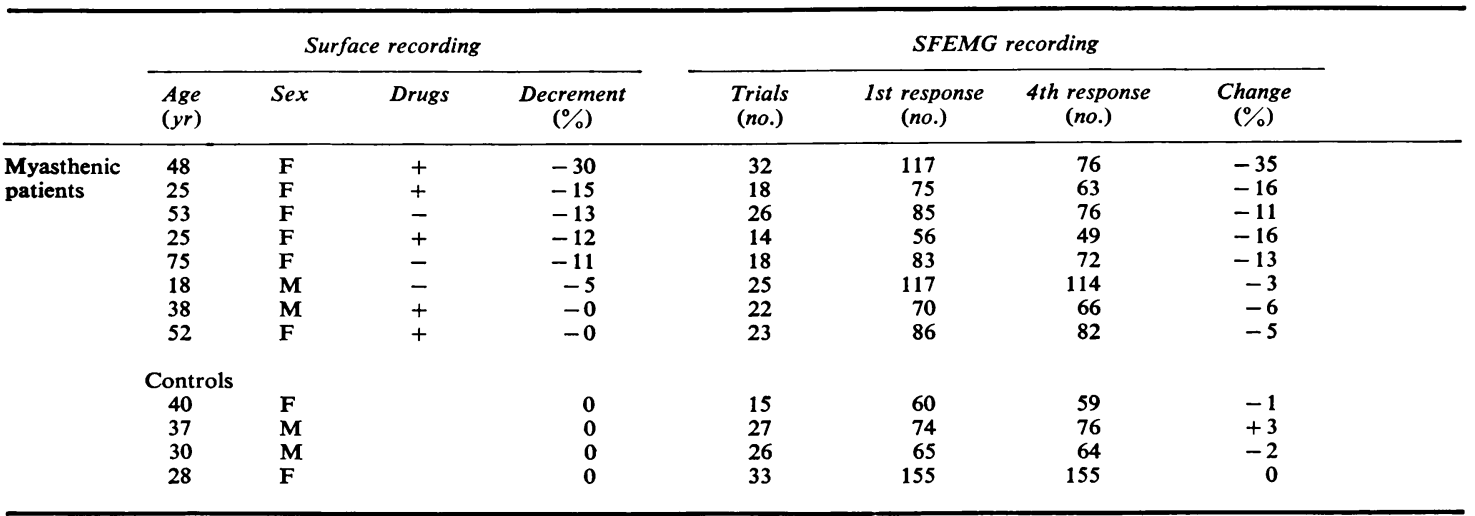


population were studied-that is, whether new spikes with different shape or latency had appeared.

SUBJECTS Four normal subjects, aged 28-40 years, with no evidence of neuromuscular disorders, were studied, as well as eight patients aged 18-75 years with myasthenia gravis. Two of the patients had only ocular symptoms and these patients, together with an additional patient, had never received therapy.

\section{RESULTS (Table)}

In normal subjects, the SFEMG findings on voluntary activity were normal and there was no decrement of evoked muscle action potential to repetitive stimulation with surface recordings. With SFEMG recordings at $2 \mathrm{~Hz}$ stimulation the fourth response averaged from $+3 \%$ to $-2 \%$

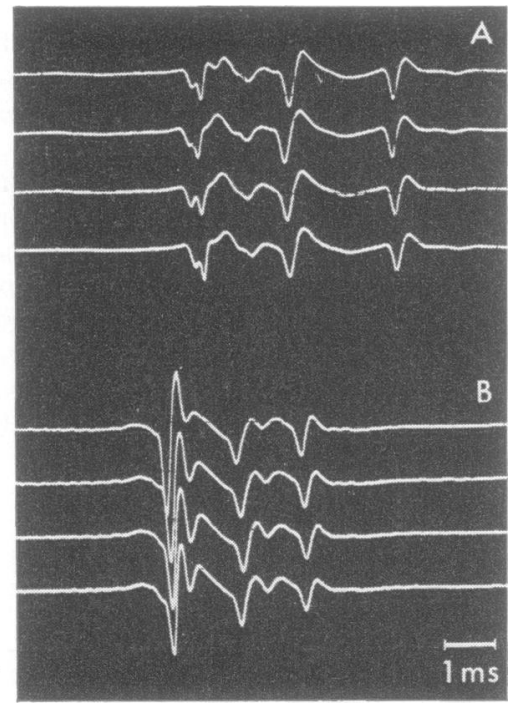

FIG. 2 Repetitive stimulation in normal subjects. In both examples a consistent response is seen of a number of single fibre action potentials.

difference in the number of components from the first. A typical response to repetitive stimulation can be seen in Fig. 2. The difference in number of spike components was usually due to slight changes in the recording position, or possibly critical submaximal stimulation. At an individual recording site one to seven distinct spike components were recorded. This signifies that usually more than one motor unit is being recorded. The reduction or increase in the number of spikes for the fourth response was rarely more than one. At times, a spike component showed an increased jitter and this was also the first one to disappear. With a slightly increased stimulus strength made after the recorded stimulus trains, these unstable potentials showed increasing stability (Fig. 1.) There was an all-or-none response concerning the single fibre components. The problems with electrode movements were minor, as judged from the relatively constant shape of the non-blocking spike components, and less than $10 \%$ of the recordings had to be discarded because of excessive electrode movements. Even at $10 \mathrm{~Hz}$ stimulation, a stable recording position could be maintained (Fig. 3). At this high rate of stimulation, there was a progressive reduction of the latency for the first few stimuli.

In the subjects with myasthenia gravis theres was a surface decrement at the ADM to supran maximal stimulation at $2 \mathrm{~Hz}$ of $30 \%, 15 \%, 13 \%$ $12 \%, 11 \%, 5 \%, 0 \%, 0 \%$ respectively. Th\& $\overrightarrow{0}$ voluntary SFEMG recordings showed increased. ov jitter in several potential pairs in the EDC and ADM in all the patients, and in six patients

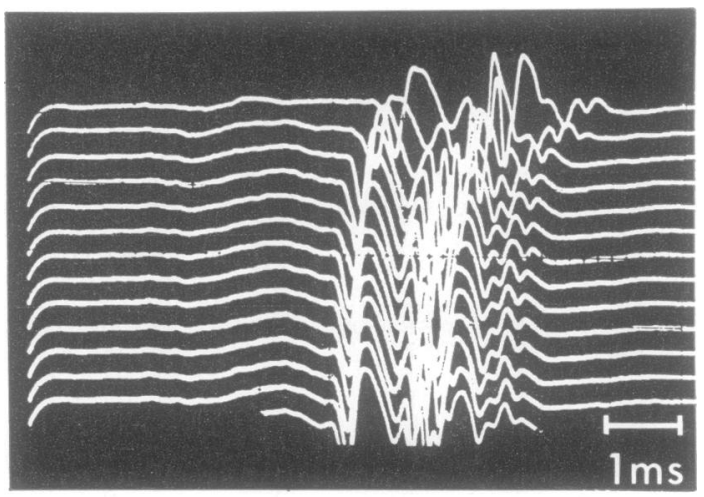

FIG. 3 Repetitive stimulation at $10 \mathrm{~Hz}$ in normal subjects. A consistent response is seen with no blocking and no change in the recording position. There is a progressive reduction in the latency of the individual components. 
impulse blockings were found in both muscles. With stimulation and SFEMG recordings the percentage reduction in number of spikes demonstrated at the fourth response was $35 \%, 16 \%$, $11 \%, 16 \%, 13 \%, 3 \%, 6 \%$, and $5 \%$ respectively. The spikes showed an all-or-none response but

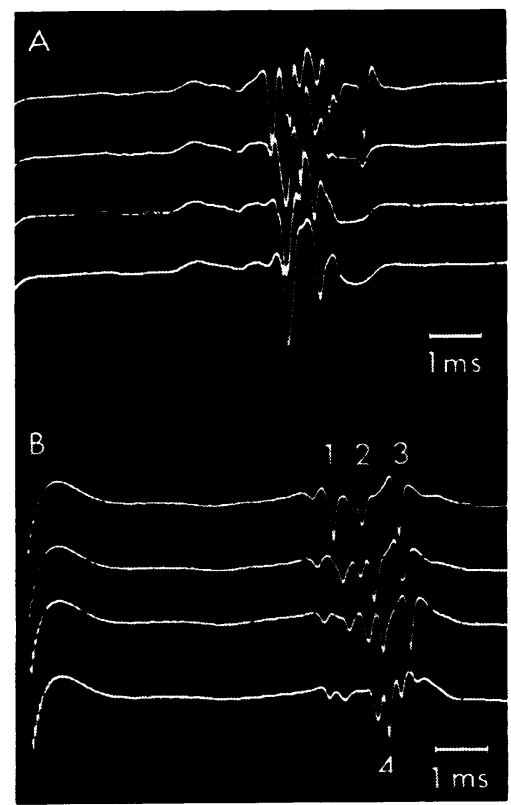

FIG. 4 Repetitive stimulation in myasthenia gravis. In A there is blocking of several components with $2 \mathrm{~Hz}$ stimulation. In B there is blocking of fibre 1 after the first stimulus, fibre 2 does not block, fibre 3 blocks only with the fourth response, and fibre 4 is recruited with the second stimulus.

the jitter of the individual components was very marked. The characteristic responses of the myasthenic group were different, even in the mild cases, from those of the normal subjects. Intermittent blocking of fibres was observed in all the myasthenic patients, particularly in the more clinically affected (see Fig. 4A). Occasionally when there was the same number of fibre action potentials in the first and fourth response this was due to the blocking of some potential and recruitment of others (see Fig. 4B). Recruitment of new fibre action potentials was seen in all patients, particularly in those who were receiving anticholinesterase therapy.

With prolonged $2 \mathrm{~Hz}$ stimulation, components absent with the fourth response occasionally reappeared at later stimuli. In addition, entirely new spike components were recruited and then persisted during a long train. Within each set of three stimulation periods, there was some constancy of the first response. The fourth responses showed a large variability relative to the first in the myasthenic group only.

\section{DISCUSSION}

It has been known for the past three decades that a decremental muscular response to repetitive stimulation can be recorded in generalized myasthenia gravis (Harvey and Masland, 1941). The clinically unaffected muscles may not show this decrement. The initial response amplitudes are usually normal but at the lower limits. The most favourable rates of stimulation are the slower ones due to less facilitation (Desmedt, 1973). In normal subjects, there may be a decrement as large as $8 \%$ (Desmedt, 1973) and 10\% has been set as the normal limit. This decrement is probably due to recording and local axonal factors and does not imply neuromuscular blocking as in myasthenia gravis. It has been shown that a stimulation rate of $100 \mathrm{~Hz}$ in normal intercostal muscle in vitro may not cause neuromuscular blocking (Elmqvist et al., 1964). In cases where voluntary SFEMG has shown definite involvement of neuromuscular transmission with increased jitter and occasional neuromuscular blockings, the decrement with usual techniques may fall within normal limits in the same muscle without provocative tests of exhaustion and ischaemia, particularly in cases of ocular myasthenia. In mild generalized cases, there is a good correlation between the presence of increased jitter and low degree of neuromuscular blocking with SFEMG and the nonsignificant decremental response to surface stimulation. In the more severely affected cases, there may be a discrepancy with a large degree of blocking but still a small decrement. The SFEMG recorded at stimulation shows that this is partially due to facilitation of previously blocked fibres. The possibility that low threshold motor units activated at voluntary contraction 
may have a lower safety factor than those motorunits activated at electrical stimulation is also raised. In such cases, the SFEMG electrical stimulation technique helps to identify the myasthenic group, although the voluntary SFEMG studies may have a higher sensitivity. In severe cases, additional information can be obtained with stimulation SFEMG relative to the voluntary activation, particularly in reference to the very abnormal neuromuscular junction. It has been shown by in vitro studies in human myasthenic intercostal muscles that some endplates of the motor units are subthreshold even with the first stimulus (Elmqvist et al., 1964). Other neuromuscular junctions have a low safety margin and will block after only a few discharges. Because of this, they can be studied only with short trains of electrical stimulation and not with the usual SFEMG technique of voluntary activation.

The good correlation of the decrement in the surface and the SFEMG recordings confirms that the surface decrement is due to impulse blockings.

In normal subjects, a reduction in number of spikes occurred at times. This was probably not due to neuromuscular failure but more likely to threshold nerve stimulation where some axons were not activated because of spontaneous fluctuations in their excitability (Blair and Erlanger, 1933). The difficulty with submaximal stimulation is that certain axons will fire intermittently in an all-or-none fashion. On increasing the stimulus strength, a constant response was obtained. In myasthenia most of the blocking components did not respond to increased stimulus strength. The problem with subthreshold stimulation could be overcome by using supramaximal stimulation so that all axons could be fully activated, but the recorded action potentials would be obscured by a mass response from many motor units as well as by gross movements of the recording electrode due to the muscular twitch. In the present technique, only minimal signs of electrode movements occurred and the amplitude of the action poten- tials from the occasional blocking muscle fibres were relatively constant when they appeared. The change in latency with rapid rates of stimulation (Fig. 3) is a physiological phenomenon mainly due to increase in muscle fibre propagation velocity (Stålberg, 1966). This corresponds to pseudofacilitation in surface responses to repetitive stimulation (Desmedt, 1973).

In conclusion, the SFEMG stimulation method offers possibilities of evaluating neuromuscular transmission of single neuromuscular junctions testing several motor units at the same time. The method allows an improved evaluation of the patient who is minimally affected with an insignificant surface decrement as well as permitting study of the severely affected neuromuscular junctions.

The investigation was supported by the Swedish Medical Research Council (Grant No. 135).

\section{REFERENCES}

Blair, E. A., and Erlanger, J. (1933). A comparison of the characteristics of axons through their individual electrical responses. American Journal of Physiology, 106, 524-564.

Desmedt, J. E. (1973). The neuromuscular disorder in myas응 thenia gravis. 1. Electrical and mechanical response to nerve stimulation in hand muscles. In New Developments inco Electromyography and Clinical Neurophysiology, vol. $1, \mathrm{~F}$ pp. 241-304. Edited by J. E. Desmedt. Karger: Basel.

Ekstedt, J. (1964). Human single muscle fiber action potentials. Acta Physiologica Scandinavica, 61. Suppl. 226.

Ekstedt, J., and Stålberg, E. (1967). Myasthenia gravis. Diagnostic aspects by a new electrophysiological method. Opuscula Medica, 12, 73-76.

Elmqvist, D., Hofmann, W. W., Kugelberg, J., and Quastel, D. M. J. (1964). An electrophysiological investigation of neuromuscular transmission in myasthenia gravis. Journal of Physiology, 174, 417-434.

Harvey, A. M., and Masland, R. L. (1941). The electromyogram in myasthenia gravis. Bulletin of the Johns Hopkins Hospital, 69, 1-13.

Stålberg, E. (1966). Propagation velocity in human muscle fibres in situ. Acta Physiologica Scandinavica, 70. Suppl. 287.

Stålberg, E., and Ekstedt, J. (1973). Single fibre EMG and microphysiology of the motor unit in normal and diseased human muscle. In New Developments in Electromyography and Clinical Neurophysiology, vol. 1, pp. 113-129. Edited by J. E. Desmedt. Kargar: Basel.

Stålberg, E., Ekstedt, J., and Broman, A. (1974). Neuromuscular transmission in myasthenia gravis studied with single fibre electromyography. Journal of Neurology, Neurosurgery, and Psychiatry, 37, 540-547. 